\title{
Uma introdução à Teoria de Landau para Líquidos de Fermi
}

\author{
An introduction to Landau's Theory of Fermi Liquids \\ Rui Aquino*10, Daniel G. Barci ${ }^{1}$ \\ ${ }^{1}$ Universidade do Estado do Rio de Janeiro, Departamento de Física Teórica, 20550-013, Rio de Janeiro, RJ, Brasil.
}

Recebido em 09 de abril de 2020. Revisado em 15 de maio de 2020. Aceito em 17 de maio de 2020.

\begin{abstract}
Apresentamos uma introdução à Teoria de Landau para Líquidos de Fermi. Procuramos evidenciar os paradigmas estabelecidos por Lev Landau nos anos 50, que têm impacto para o desenvolvimento de áreas de pesquisa até os dias atuais. Por sua vez, argumentamos que é possível o ensino dessa teoria em cursos de graduação em física, tendo um impacto positivo na construção do conhecimento de profissionais de áreas como Estado Sólido e Elétrons Fortemente Correlacionados. A apresentação é construtiva, autocontida e voltada para alunos de graduação e pós graduação em Física. A modo de exemplo, estudamos a propagação de ondas em um modelo simplificado de líquido de Fermi bidimensional. Relacionamos o resultado com um dos cálculos mais conhecidos de Landau, as excitações de "som zero".
\end{abstract}

Palavras-chave: Líquidos de Fermi, Sistemas Fortemente Correlacionados.

\begin{abstract}
We present an introduction to Landau's Theory of Fermi Liquids. We seek to highlight the paradigms established by Lev Landau in the 1950s, which influence the development of current research areas. In turn, we argue that it is possible to teach this theory in physics undergraduate courses, having a positive impact on the knowledge building of professionals specialized in areas such as Solid State and Strongly Correlated Electrons. The presentation is constructive, self-contained and aimed at Physics undergraduate and graduate students. As an example, we study wave propagation in a simplified two-dimensional Fermi liquid model. The output is related with one of Landau's best known results, the "zero sound" excitation.
\end{abstract}

Keywords: Fermi Liquids, Strongly Correlated Systems.

\section{Introdução}

O estudo da matéria em condições extremas de temperatura, pressão e campos magnéticos é uma área fascinante da física. Quando a matéria é submetida a temperaturas muito baixas, ou pressão e densidade muito altas, as propriedades quânticas se fazem evidentes afetando mesmo grandezas macroscópicas como o calor específico, condutividade, suscetibilidade magnética etc. Por experiência, sabemos que a maior parte dos líquidos solidificam, quando resfriados suficientemente à pressão atmosférica. Porém, existem substâncias como o $H e$, o qual continua líquido, mesmo a temperaturas muito perto do zero absoluto. Nessas condições, a teoria clássica dos líquidos não é aplicável, surgindo assim o conceito de "líquido quântico". Embora o $\mathrm{He}$ tenha sido o primeiro líquido quântico descoberto, o conceito não está restrito a esse material. O comportamento eletrônico da maioria dos metais pode ser considerado como um líquido quântico. Ainda, o núcleo de estrelas, sob determinadas condições, também podem ser considerado um líquido quântico. Mas recentemente, com o desenvolvimento de novas técnicas de resfriamento atômico e molecular, muitos sistemas gasoso

*Endereço de correspondência: ruiaquino96@gmail.com em condições normais, entram na fase líquida quântica quando resfriados a temperaturas extremamente baixas.

De uma forma geral, a teoria que melhor descreve a matéria nessas condições extremas é a Mecânica Estatística Quântica. Essa teoria combina o tratamento estatístico de um número muito grande de partículas em equilíbrio termodinâmico com a natureza quântica da matéria. Sabemos que existem dois tipos de propriedades estatísticas bem diferentes. Elas são chamadas de estatística bosônica e fermiônica. Os nomes adotados são uma homenagem aos físicos Enrico Fermi e Satyendra Bose devido à descoberta destas propriedades. Dessa forma, chamamos bósons às partículas que satisfazem a estatística bosônica e férmions aquelas que satisfazem a estatística fermiônica. Existe um teorema muito interessante chamado teorema de spin-estatística [1,2], que associa os bósons às partículas de spin inteiro, e os férmions às partículas de spin semi-inteiro. Mesmo sem entrar nos detalhes da demonstração, é curioso como duas propriedades completamente diferentes podem estar relacionadas; enquanto que o spin é uma propriedade intrínseca de uma partícula, sua estatística é uma propriedade de um conjunto muito grande de partículas (ensemble). Cabe destacar que as propriedades estatísticas aqui mencionadas são próprias de um 
espaço tridimensional. Os sistemas bidimensionais são muito mais ricos, admitindo um número muito maior de propriedades estatísticas quânticas. Dessa forma, podemos concluir que existem dois tipos principais de líquidos quânticos. Os compostos por férmions, aos quais chamamos de líquidos de Fermi e os compostos por bóson, chamados líquidos ou condensados de Bose.

No presente artigo vamos nos concentrar nos líquidos de Fermi. $\mathrm{O}^{3} \mathrm{He}$ é um isótopo do $H e$ que tem spin semi-inteiro e foi o primeiro líquido de Fermi estudado. Por outro lado, quase todos os metais são líquidos de Fermi, já que estão compostos essencialmente por elétrons. Ainda, o núcleo de estrelas de nêutrons pertencem a essa categoria, dado que o nêutron é uma partícula de $\operatorname{spin} 1 / 2$.

Embora, formalmente, a mecânica estatística quântica possa descrever as propriedades de um líquido de Fermi, na prática, é muito difícil atacar sistemas realistas com esse formalismo, já que as interações fortes fazem inadequado qualquer aproximação perturbativa. Por esse motivo, é conveniente dispor de teorias fenomenológicas que possam descrever o fenômeno com o menor número de parâmetros arbitrários. Esses parâmetros, a ser determinados experimentalmente, são o preço a pagar por não dispor de uma teoria microscópica que possa ser tratada matematicamente. Neste artigo, faremos uma introdução à "Teoria de Landau para Líquidos de Fermi", a qual teve um enorme sucesso na descrição qualitativa e quantitativa desse estado da matéria.

Por outro lado, um dos principais problemas em aberto na área de sistemas fortemente correlacionados e que tem um interesse crescente na comunidade de Física da Matéria Condensada, é entender os estados da matéria denominados "Non-Fermi Liquids". Essa denominação é usada para descrever compostos metálicos que não seguem os paradigmas estabelecidos pela teoria de Landau para líquidos de Fermi nos anos 50. Fica evidente que a teoria de Landau também serve de ponto de partida para o estudo de assuntos que estão em foco no que há de mais avançado em pesquisa. Alguns desses assuntos têm sido agraciados recentemente com prêmios, como a descoberta de fases exóticas do $\mathrm{He}$ que rendeu o prêmio Nobel a A. Legget no ano de 2003, e teorias sobre suas propriedades topológicas que foram reconhecidas em 2016 com o prêmio Nobel à D. Haldane, D. Thouless e J. Kosterlitz.

Apesar de seu caráter fundamental no desenvolvimento epistemológico da área de sistemas fortemente correlacionados, é intrigante que essa teoria não seja ensinada em cursos de graduação. Afirmamos isso pois o nível de desenvolvimento da Teoria de Landau é tal que alunos poderiam aprendê-la na mesma época em que estivessem cursando disciplinas como Estrutura da Matéria, visto que um conhecimento mais profundo de Física Estatística e Quântica não são estritamente necessários para o aprendizado do assunto. Também não vemos com frequência essa teoria sendo ensinada em cursos de pós graduação.
Embora sem a certeza do porquê dessa ausência, podemos ao menos afirmar, que em ambos os currículos, os conceitos que Landau estabeleceu são ensinados de forma diluída em cursos de Estado Sólido e Matéria Condensada. Longe de problematizar a estrutura curricular dos cursos de Física brasileiros, temos como interesse nesse trabalho sugerir um olhar mais cuidadoso, mesmo que introdutório, sobre um assunto de vital importância histórica e de pesquisa atual. De fato, ainda hoje, diversos trabalhos de alto nível em Líquidos de Fermi, assim como em assuntos que se utilizam dos conceitos introduzidos nessa teoria, são publicados frequentemente na literatura especializada.

Com o objetivo de explicar a teoria de Landau de forma mais autoconsistente possível, organizamos o artigo da seguinte forma, partindo do conceito de um Gás de Fermi na seção 2, explicamos os conceitos e fundamentos da Teoria de Líquidos de Fermi nas sessões 3 e 4 . Finalizamos então o trabalho entendendo como trabalhar com dinâmica em Líquidos de Fermi e analisando a solução mais famosa de Landau, o "som zero" de Landau, na seção 5

\section{O gás de Fermi}

\subsection{O estado fundamental de um gás de Fermi e o principio de exclusão de Pauli}

O primeiro passo para entender as propriedades físicas de um líquido de Fermi é a descrição de um sistema muito mais simples: o gás ideal de Fermi. Esse sistema é composto de $\mathrm{N}$ férmions não interagentes. Embora o conceito de férmions apareça no estudo da mecânica quântica de partículas idênticas, vamos mostrar que o única propriedade realmente necessária para a descrição do fluido fermiônico é o principio de exclusão de Pauli. Este principio estabelece que duas partículas fermiônicas não podem ocupar um mesmo estado quântico. Este principio é o responsável, por exemplo, da distribuição e organização dos elementos na tabela periódica de Mendeleev. No nosso caso, ele vai dar lugar à formação do segundo conceito importante da nossa apresentação: a superfície de Fermi.

A energia de uma partícula livre é dada por,

$$
\epsilon_{\boldsymbol{k}}=\frac{|\boldsymbol{k}|^{2}}{2 m}
$$

onde $\boldsymbol{k}$ é o momento da partícula (ao longo do artigo usamos a notação "negrita"para indicar uma quantidade vetorial e um sistema de unidades no qual a constante de Planck $\hbar=1$ ). Se considerarmos o volume do espaço infinito, o espaço de momentos é continuo. Porém, se trabalharmos num volume finito $V$, os momentos serão discretizados. Esta diferença não é importante e usaremos ambos esquemas indistintamente dependendo da conveniência. Uma representação gráfica da equação (1) em duas dimensões pode ser apreciada na figura 1 . 


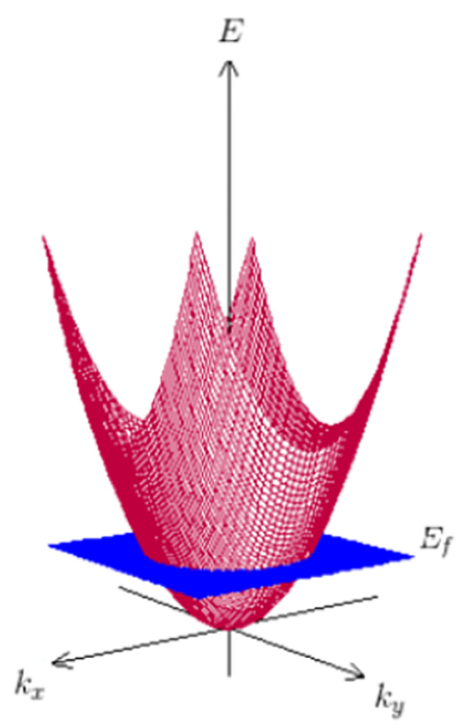

Figura 1: Relação de dispersão. Energia em função de momento dado pela equação (1) em duas dimensões, $\boldsymbol{k}=\left(k_{x}, k_{y}\right)$

Uma pergunta importante é qual o estado de menor energia de um sistema de $N$ férmions com a relação de dispersão dada pela equação (1). Este estado é chamado de estado fundamental do sistema. Se considerarmos os férmions como elétrons, ou seja, partículas de spin $1 / 2$, cada estado do sistema é caracterizado por dois números quânticos $(\boldsymbol{k}, \sigma)$, onde $\boldsymbol{k}$ é o momento e $\sigma=$ $\pm 1 / 2$ é a componente $z$ do spin, que pode tomar dois valores. Portanto, devido ao princípio de exclusão de Pauli, apenas podem existir duas partículas com o mesmo valor do momento, uma com spin $\sigma=1 / 2$ e a outra com $\sigma=-1 / 2$. Dessa forma, as $N$ partículas ficarão distribuídas ocupando os valores de momento que têm a menor energia possível. O valor da energia da partícula mais energética é chamada de energia de Fermi, $\epsilon_{F}$. Esse conceito pode ser visualizado na figura 1. As partículas preenchem os estados de menor energia até o plano $\epsilon_{F}$ indicado na figura. Desta forma, os estados com energia $\epsilon(\boldsymbol{k})<\epsilon_{F}$ são preenchidos por partículas, enquanto que os estados com $\epsilon(\boldsymbol{k})>\epsilon_{F}$ estão vazios. A equação

$$
\epsilon\left(\boldsymbol{k}_{\boldsymbol{F}}\right)=\epsilon_{F}
$$

define a chamada superfície de Fermi. Ela é formada pelos valores de momento $\boldsymbol{k}=\boldsymbol{k}_{F}$ que satisfazem a equação (2). A representação gráfica da equação (2), nada mais é que a interseção entre o paraboloide e o plano indicado na figura 1 . O resultado é um circulo de raio $k_{F}$ indicado na figura 2

$k_{F}$ é chamado de momento de Fermi. De tal forma que o interior do circulo $|\boldsymbol{k}|<k_{F}$ representa estados preenchidos com partículas, enquanto que o exterior $|\boldsymbol{k}|>$ $k_{F}$ representa estados vazios. A linha divisória $|\boldsymbol{k}|=k_{F}$ é precisamente a superfície de Fermi. Note que em duas dimensões, a "superfície" de Fermi é na verdade uma linha. O mesmo raciocínio em três dimensões leva a

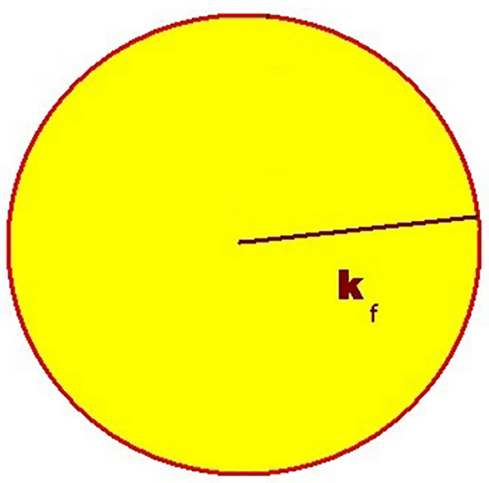

Figura 2: Superfície de Fermi de um sistema bidimensional de fermions livres. $k_{F}$ é o chamado momento de Fermi. Os estados dentro do círculo, $|\boldsymbol{k}| \leq k_{F}$ estão completamente preenchidos com partículas. Por outro lado, os estados fora do círculo, $\mid \boldsymbol{k}>$ $k_{F} \mid$ estão vazios.

uma esfera de Fermi, por este motivo é o nome geral de "superfície".

Podemos descrever os mesmos conceitos de forma matemática introduzindo uma função chamada de número de ocupação do estado fundamental, definida como

$$
n_{\mathbf{k}, \sigma}^{0}= \begin{cases}1, & \epsilon \leqslant \epsilon_{F} \\ 0, & \epsilon>\epsilon_{F}\end{cases}
$$

Essa função apenas indica que existe uma partícula para cada valor do spin $\sigma$, se a energia for menor que a energia de Fermi, e não existem partículas no caso contrário. O sobre-índice 0 denota que estamos no estado fundamental, ou seja, o estado de menor energia possível. Na figura 3 essa função pode ser apreciada como função de $|\boldsymbol{k}|$. A descontinuidade de $n_{|\boldsymbol{k}|, \sigma}^{0}$ em $|\boldsymbol{k}|=k_{F}$ define a localização da superfície de Fermi.

Essa função pode ser usada para expressar o número de partículas e a energia total do estado fundamental do gás de Fermi da seguinte forma,

$$
\begin{aligned}
N & =\sum_{\boldsymbol{k}, \boldsymbol{\sigma}} n^{0}{ }_{\boldsymbol{k}, \boldsymbol{\sigma}} \\
E^{0} & =\sum_{\mathbf{k}, \sigma} \frac{\mathbf{k}^{2}}{2 m} n_{\mathbf{k}, \sigma}^{0}
\end{aligned}
$$

onde assumimos que os valores de $\boldsymbol{k}$ são discretos. Estas equações representam uma relação entre o momento de Fermi $k_{F}$, o número de partículas total $N$ e a energia

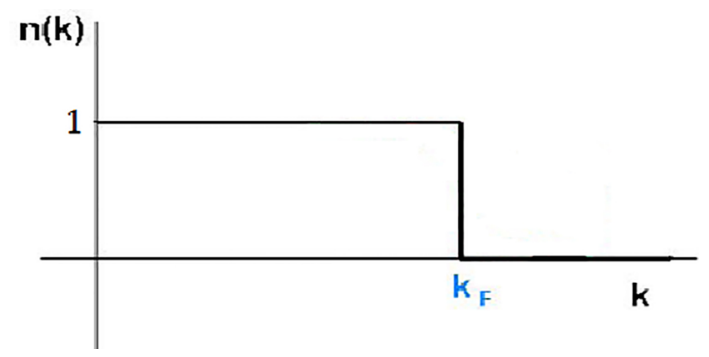

Figura 3: Número de ocupação por spin no estado fundamental, dado pela equação (3) 
total do estado fundamental $E^{0}$. Para mostrar essa relação de forma explícita temos que calcular as somas nas equações (4) e (5). Para isto, consideramos que no limite de volume muito grande o espectro é essencialmente contínuo, podemos então substituir

$$
\sum_{\boldsymbol{k}} \rightarrow V \int \frac{d^{D} \boldsymbol{k}}{(2 \pi)^{D}}
$$

onde $D$ é o número de dimensões do espaço, e $V$ o volume $D$-dimensional. O cálculo das integrais pode ser feito facilmente em coordenadas esféricas $D$-dimensional, aproveitando o fato que os integrandos tem simetria esférica, isto é, dependem apenas do $|\boldsymbol{k}|$, não dependendo dos ângulos. Temos então,

$$
\begin{aligned}
N & =V \sum_{\sigma} \int \frac{d^{D} \boldsymbol{k}}{(2 \pi)^{D}} n_{\sigma}^{0}(\boldsymbol{k})=\frac{V \Omega_{D}}{(2 \pi)^{D}} \int d k k^{D-1} \sum_{\sigma} n_{\sigma}^{0}(k) \\
& =\frac{2 V \Omega_{D}}{(2 \pi)^{D}} \int_{0}^{k_{F}} d k k^{D-1}=\frac{2 \Omega_{D}}{(2 \pi)^{D} D}\left(V k_{F}^{D}\right)
\end{aligned}
$$

$\mathrm{Na}$ primeira linha usamos o fato de o integrando ser invariante perante rotações. $\Omega_{D}=2 \pi^{D / 2} / \Gamma(D / 2)$, onde $\Gamma(D / 2)$ é a função Gamma, é a contribuição angular do volume de integração; é a superfície de uma hyper-efera $D$-dimensional de raio um. Na segunda linha, usamos o fato que $n_{\boldsymbol{k}, \sigma}=\Theta\left(k_{F}-|\boldsymbol{k}|\right)$, onde $\Theta$ é a função degrau, ou distribuição de Heaviside. O fator 2 vem da degenerescência de spin. O resultado final foi arrumado de tal forma que fique evidente que é adimensional.

De forma análoga podemos calcular também energia total no estado fundamental $E^{0}$, equação(5). Encontramos a expressão,

$$
E^{0}=\frac{2 V}{(2 \pi)^{D}} \frac{\Omega_{D}}{2 m} \frac{k_{F}^{D+2}}{D+2}
$$

Um resultado interessante, que pode ser facilmente deduzido a partir dessas relações, é que a energia de Fermi de um gás ideal é exatamente igual ao potencial químico, $\epsilon_{F}=\mu$. O potencial químico é definido como a energia necessária para introduzir uma nova partícula em um sistema que tem originalmente $N$ partículas. Isto é $\mu=E^{0}(N+1)-E^{0}(N)$. No limite de $N$ muito grande, esta expressão é equivalente a $\mu=\partial E^{0} / \partial N$. Usando a regra da cadeia, e as expressões equações (7) e 8 temos que,

$$
\begin{aligned}
\mu & =\frac{\partial E_{0}}{\partial k_{F}} \frac{\partial k_{F}}{\partial N}=\left(\frac{2 V}{(2 \pi)^{D}} \frac{\Omega_{D}}{2 m} k_{F}^{D+1}\right)\left(\frac{(2 \pi)^{D}}{2 V} \frac{1}{\Omega_{D}} k_{F}^{1-D}\right) \\
& =\frac{k_{F}^{2}}{2 m}=\epsilon_{F} .
\end{aligned}
$$

Logo, concluímos que a energia no nível de Fermi é dada pelo potencial químico. Veremos que este resultado pode ser modificado pelas interações entre partículas e pela temperatura.
Muitas propriedades termodinâmicas e de transporte não dependem apenas do estado fundamental do sistema mas também de estados excitados. Portanto, é interessante investigar como se modifica a energia quando a distribuição de partículas se afasta do estado fundamental. Para um gás ideal, podemos definir em geral a energia de qualquer distribuição de partículas como

$$
E\left[n_{\mathbf{k}, \sigma}\right]=\sum_{\mathbf{k}, \sigma} \frac{\mathbf{k}^{2}}{2 m} n_{\mathbf{k}, \sigma}
$$

Note que essa expressão é equivalente à equação (5). Porém, a distribuição $n_{\mathbf{k}, \sigma}$ é em princípio arbitrária, não necessariamente a correspondente ao estado fundamental. É interessante reescrever esta expressão colocando em evidência a energia do estado fundamental. Para isto, definimos

$$
\delta n_{\mathbf{k}, \sigma}=n_{\mathbf{k}, \sigma}-n_{\mathbf{k}, \sigma}^{0}
$$

Onde $\delta n_{\mathbf{k}, \sigma}$ é o desvio que a distribuição perturbada teve em comparação ao seu estado fundamental $n_{\mathbf{k}, \sigma}^{0}$. Podemos assim escrever a energia do sistema excitado como:

$$
E\left[\delta n_{\mathbf{k}, \sigma}\right]=E_{0}+\sum_{\mathbf{k}, \sigma} \frac{\mathbf{k}^{2}}{2 m} \delta n_{\mathbf{k}, \sigma}
$$

Esta expressão será muito útil na hora de introduzir interações para a descrição de um líquido de Fermi como veremos nas próximas sessões. Uma observação importante é que a partir da equação 12 , pode ser definida relação de dispersão de uma partícula derivando funcionalmente a energia total em relação ao desvio da distribuição de partículas. Isto é,

$$
\epsilon(\mathbf{k})=\frac{\delta E\left[\delta n_{\mathbf{k}, \sigma}\right]}{\delta \delta n_{\mathbf{k}}}=\frac{\mathbf{k}^{2}}{2 m}
$$

Embora este resultado seja o esperado, veremos que ele é modificado de forma não trivial na presença de interações.

\subsection{Termodinâmica do Gás de Fermi}

A rigor, as propriedades termodinâmicas de um gás de Fermi podem ser obtidas a partir da Mecânica Estatística Quântica. A partir da Grande Função de Partição, podem ser calculados potenciais termodinâmicos e, a partir destes os observáveis relevantes. Porem, neste artigo, podemos fazer uma analise simplificada já que apenas estamos interessados nas propriedades de temperaturas extremamente baixas. Para isto, é suficiente tomar um resultado da Mecânica Estatística que fornece o número de ocupação médio em função da temperatura. Ele pode ser escrito como,

$$
\left\langle n_{\mathbf{k}, \sigma}\right\rangle=\frac{1}{1+e^{\left(\epsilon_{\mathbf{k}}-\mu\right) / k_{B} T}}
$$

Onde $\epsilon_{\mathbf{k}}$ é a relação de dispersão de uma partícula; no nosso caso é dado pela equação (1). $k_{B}$ é a constante de 
Boltzmann e $T$ a temperatura absoluta do sistema. A equação (14) é chamada de distribuição de Fermi-Dirac.

É interessante observar que existe uma escala particular onde a distribuição muda o regime qualitativamente. Isto acontece quando a energia térmica é da ordem da energia de Fermi, $k_{B} T=\epsilon_{F}$. Isto define a chamada temperatura de Fermi $T_{F}=\epsilon_{F} / k_{B}$. Para $T>>T_{F}$, o gás se comporta como um gás clássico. $\mathrm{O}$ regime $T<T_{F}$, é chamado de gás fermiônico degenerado. Já o limite em que $T \rightarrow 0,\left\langle n_{\mathbf{k}, \sigma}\right\rangle \rightarrow n_{\mathbf{k}, \sigma}^{0}$, recebe o nome de gás completamente degenerado.

É instrutivo observar alguns valores da temperatura de Fermi para diferentes sistemas. Por exemplo, a temperatura de Fermi do cobre é $T_{F}=8.16 \times 10^{4} \mathrm{~K}$. Ou seja, muito acima da temperatura de fusão do cobre. Isso significa que os elétrons num fio de cobre a temperatura ambiente podem ser considerados como um gás de Fermi degenerado com muito boa aproximação. De fato, na maioria dos metais, a temperatura de Fermi é da ordem de $10^{4} \mathrm{~K}$. Por outro lado, a temperatura típica de uma estrela "anã branca" é de $T \sim 10^{7} \mathrm{~K}$. Porém, a temperatura de Fermi dessa estrela é da ordem de $T_{F} \sim 10^{9} \mathrm{~K}$, o que significa que o gás de elétrons dentro da estrela é "muito frio", já que $T<<T_{F}$, e pode ser considerado como uma gás de Fermi degenerado.

Um ponto importante é observar as características dos desvios

$$
\delta n_{\boldsymbol{k}, \sigma}(T)=\left\langle n_{\mathbf{k}, \sigma}\right\rangle-n_{\mathbf{k}, \sigma}^{0}
$$

como função da temperatura. Para $T<<T_{F}$, essa distribuição é fortemente concentrada ao redor de $k_{F}$, sendo praticamente zero para qualquer outro valor do momento. Este comportamento é ilustrado na figura 4. Nessa figura desenhamos $\left|\delta n_{\boldsymbol{k}, \sigma}(T)\right|$ dado pela equação (15) em função do momento, reescalado com o valor do momento de Fermi, $k / k_{F}$. Em particular, fixamos os valores $\epsilon_{F}=1 / 2$ e $k_{B} T=0.01$.

A interpretação física é muito clara. Uma perturbação térmica ao estado fundamental conserva o número de

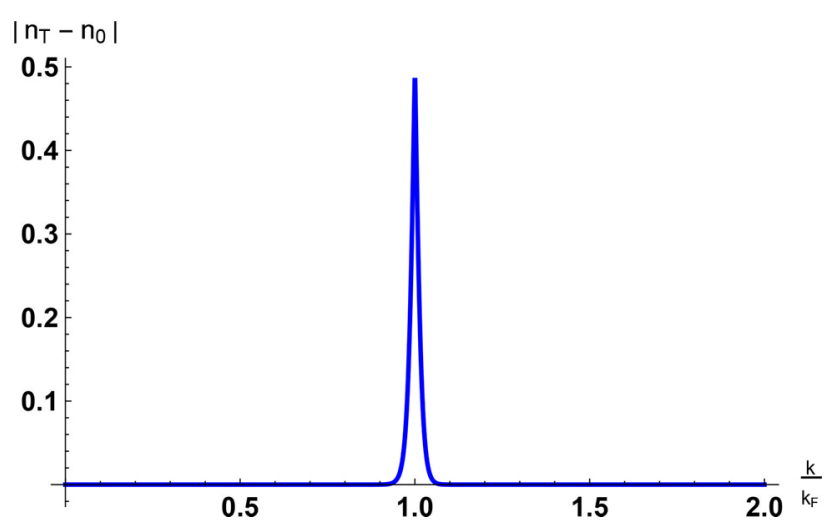

Figura 4: $\left|\delta n_{\boldsymbol{k}, \sigma}(T)\right|$ dada pela equação (15) em função de $k / k_{F}$. Os parâmetros foram fixados nos valores $\epsilon_{F}=1 / 2 \mathrm{e}$ $k_{B} T=0.01$. partículas. Por este motivo, qualquer estado excitado trata-se de uma excitação partícula-buraco. Isto significa que uma partícula é removida com energia menor que a energia de Fermi, sendo promovida a um estado com energia maior que a de Fermi. Porém, como a energia térmica disponível é muito pequena $k_{B} T<<\epsilon_{F}$, apenas partículas muito perto da superfície de Fermi podem participar dessas excitações. Este é o motivo pelo qual os desvios do estado fundamental estão tão concentrados ao redor do momento de Fermi. Usando apenas esse fato, e a equação 12 , pode ser calculada qualquer quantidade termodinâmica a baixas temperaturas.

Por exemplo, a equação de estado de um gás de Fermi ideal à $T<<T_{F}$ é dado por 3 :

$$
P V=\frac{2}{5} N k_{B} T_{F}
$$

Onde $P$ é a pressão, $V$ o volume ocupado por esse gás. Este resultado é típico de um gás de Férmions, sendo muito diferente do resultado para um gás ideal clássico. Isto é, à temperatura zero existe uma pressão, já que $P V=$ constante. Por outro lado, no caso clássico, $P V \sim T$, sendo que a pressão vai para zero de forma linear com a temperatura. A razão fundamental para existência de pressão a temperatura zero é o princípio de exclusão de Pauli que não permite colocar mais de uma partícula no mesmo estado quântico. Vemos portanto que a mecânica quântica se manifesta em uma quantidade macroscópica como a pressão em um gás.

Na mesma aproximação podemos escrever o calor específico $C_{V}$ para o gás de Fermi degenerado como:

$$
C_{V}=\frac{\pi^{2}}{2} N k_{B} \frac{T}{T_{F}}
$$

O comportamento linear com a temperatura é característica do gás de férmions. O comportamento $C_{V} \rightarrow 0$ para $T \rightarrow 0$ é compatível com a terceira lei da termodinâmica. Sabe-se que para altas temperaturas, no regime não degenerado, $C_{V} / N k_{B} \rightarrow 3 / 2$, como mostra a Figura 5

Outra quantidade termodinâmica importante que permite testar a aproximação do gás de Fermi em um sistema

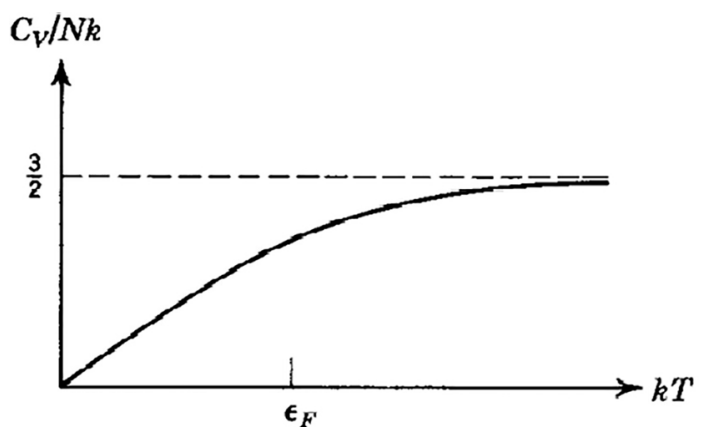

Figura 5: Calor específico de um gás de Fermi em função da temperatura, $C_{V} / N k_{B} \times k_{B} T$. O comportamento linear perto da origem é característico de um sistema fermiônico. 
real é o chamado Raio de Wilson que é definido como

$$
R_{W}=\frac{4}{3}\left(\frac{\pi k_{B}}{g \mu_{B}}\right)^{2} \frac{\chi}{C_{V} / T}
$$

onde $\chi$ é a suscetibilidade magnética, $g$ é o fator de Landé e $\mu_{B}$ é o magneton de Bohr. Calculando está quantidade nas mesmas aproximações $T<<T_{F}$, obtemos $R_{W}=1$. Portanto, a partir de dois observáveis , $C_{V}$ e $\chi$, pode ser determinado o valor de $R_{W}$. Qualquer desvio da unidade nos descreve um sistema correlacionado. Quanto maior o desvio, maior a necessidade de melhorar nossa aproximação do gás ideal, levando em conta interações, assunto que será abordado a seguir.

\section{Efeito das Interações}

A descontinuidade observada na função distribuição $n_{\mathbf{k}}^{0}$, para o sistema não interagente no estado fundamental à $\mathrm{T}=0$, Figura 3 , denota a existência da superfície de Fermi em $|\mathbf{k}|=k_{F}$. Considerar um sistema interagente leva ao questionamento sobre qual o efeito das interações e se a superfície de Fermi continua a existir.

Uma intuição ingênua pode dizer que uma interação forte causa colisões, jogando partículas com $|\mathbf{k}|<k_{F}$ para algum estado desocupado de momento $|\mathbf{k}|>k_{F}$, desmanchando assim a superfície de Fermi [4]. Porém, no geral, isso não acontece. Diversos experimentos 5. mostram que a superfície de Fermi continua existindo em sistemas fortemente correlacionados. O efeito das interações é deformar a distribuição de partículas no estado fundamental como mostra a Figura 6. Nota-se que a deformação principal, em relação à distribuição do gás ideal, ocorre muito perto do momento de Fermi, porém, ainda existe uma descontinuidade em $|\mathbf{k}|=k_{F}$, o que sinaliza a existência da superfície de Fermi.

Note que, embora a descontinuidade da função de distribuição ainda exista, seu valor é diminuído pelas interações. Para um gás ideal, o valor da descontinuidade é sempre a unidade, já que a diferença do interior ao exterior da superfície de Fermi é sempre de uma partícula. Costuma-se denotar este fato $\operatorname{com} Z=1$, onde $Z$ é o valor da descontinuidade (o tamanho do pulo na função

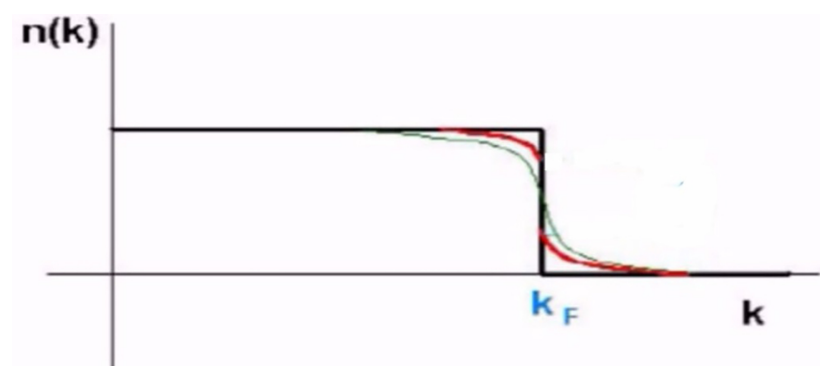

Figura 6: Estado fundamental do sistema interagente. O efeito das interações é deformar a distribuição de partículas perto da superfície de Fermi, porém mantendo a descontinuidade da função de distribuição). O valor $Z<1$, (a $T=0$ ), denota a existência de interações no sistema. Ainda, existem materiais exóticos nos quais $Z \rightarrow 0$, tendo uma distribuição de partículas contínua. Porém, esses casos não são considerados líquidos de Fermi, recebendo a denominação geral (em língua inglesa) de "non-Fermi liquids". Esses casos especiais de líquidos quânticos, embora sejam sumamente interessantes, não serão considerados no presente artigo.

\subsection{Princípio adiabático, quase-partículas e quase-buracos}

Sendo uma teoria fenomenológica, a Teoria de Landau se baseia em assumir certos princípios, porém, menos intuitivos. O principal deles, é o chamado "princípio adiabático". Segundo este princípio, se assume que ao "ligar" a interação entre os férmions de forma "suave", os estados quânticos do sistemas ou, os autoestados $\left|\psi_{i}\right\rangle_{\text {não-interagente }}$ do gás de Fermi ( o índice i representa cada nível de energia) geram todos os autoestados $\left|\psi_{i}\right\rangle_{\text {interagente }}$ do líquido de Fermi no estado fundamental [6]. Nota-se que nada garante que todos os estados serão gerados, sendo assim importante para a consistência da teoria essa correspondência um-a-um entre os estados do gás, e o sistema interagente. Na literatura [7,8], define-se o sistema que tem essa correspondência como Líquido de Fermi Normal.

A forma com que temos que conectar os autoestados é assumida como sendo adiabática, ou infinitamente lenta, sem causar uma transição de fase. Assim, todo o espectro do líquido no estado fundamental se mantém similar ao do gás de Fermi. Outra forma de visualizar esta ideia é pensar que o espectro do líquido quântico se obteria mediante deformações contínuas dos níveis de energia do gás ideal 8].

A primeira conclusão que pode-se chegar ao entender o princípio adiabático de Landau é que ao "ligarmos" a interação, ainda tem-se uma espécie de gás, visto que temos estados similares ao do gás de Fermi. Essa conclusão não é de todo errada. Pensemos por um instante o que acontece quanto tentamos introduzir mais uma partícula em um gás ideal que tem originalmente $N$ partículas. Como no gás ideal as partículas não interagem, a única coisa que vai acontecer é uma pequena modificação no nível de Fermi. Se $N$ for muito grande, essa modificação é imperceptível. Se fizermos a mesma coisa no sistema interagente, o efeito da nova partícula será rearranjar a distribuição de partículas no estado fundamental, já que ela está interagindo, a princípio, com todas as partículas no interior da superfície de Fermi. Por sua vez, as partículas do "mar de Fermi" modificam a energia da nova partícula introduzida. Se produz então um estado composto pela partícula e a "nuvem" de interações produzidas pelo rearranjo do mar de Fermi. Este estado composto foi chamado de quase-partícula. A quase-partícula tem essencialmente os mesmos números quânticos que uma partícula normal; isto é carga $e, \operatorname{spin} 1 / 2$. Tendo uma massa efetiva $\mathrm{m}^{*}$ modificada 
pelas interações. Descreve-se assim um sistema de várias partículas fortemente interagente através de um gás de constituintes fictícios fracamente interagentes, chamadas de quase-partículas.

Mesmo tendo estabelecido a correspondência um-a-um entre o gás de Fermi e o Líquido de Fermi, é importante esclarecer que usar a definição de quase-partículas se mostra adequado quando estas estão somente bem próximas da superfície de Fermi. Isto porque as quase-partículas não são estáveis. Dado que elas estão em interação com o mar de Fermi, se elas tiverem uma energia muito maior que $\epsilon_{F}$, poderiam decair em modos coletivos, que não são outra coisa senão excitações partícula-buraco, ou flutuações da superfície de Fermi. O tempo de vida das quase-partículas é muito grande perto da superfície de Fermi e diminui com o aumento da energia. Dessa forma, se o tempo em que se liga a interação é mais longo que o tempo de vida das quase-partículas, o sistema decai muito antes de alcançar-se o valor de interação necessário para a descrição do sistema. Por outro lado, se a interação é ligada muito rápido, o processo não é mais adiabático e pode ocorrer uma transição de fase 8].

A existência da superfície de Fermi na presença de interações foi assumido por Landau para a construção da teoria de líquidos de Fermi. Por outro lado, a partir do princípio adiabático, pode ser concluído que o volume da superfície de Fermi não deveria ser modificado na presença de interações, dado que ele é proporcional à densidade de partículas. Este fato, foi demonstrado mais tarde de forma perturbativa por Luttinger [9], e Migdal [10] e é conhecido na literatura como o "Teorema de Luttinger".

\subsection{Funcional de Energia}

A partir do princípio adiabático de Landau, podemos formular a teoria por analogia com o gás de Fermi descrito nas sessões anteriores. Por analogia com a equação (11) podemos escrever,

$$
\delta n_{\mathbf{k}, \sigma}=n_{\mathbf{k}, \sigma}-n_{\mathbf{k}, \sigma}^{0}
$$

Onde agora, $\delta n_{\mathbf{k}, \sigma}, n_{\mathbf{k}, \sigma}$ e $n_{\mathbf{k}, \sigma}^{0}$ correspondem ao desvio, à distribuição no estado excitado e à distribuição em seu estado fundamental de quase-partículas.

A energia das excitações pode ser considerada como um funcional $E\left[\delta n_{\mathbf{k}, \sigma}\right]$. Supondo que $\delta n_{\mathbf{k}, \sigma}$ é concentrada ao redor da superfície de Fermi, podemos expandir esse funcional em série de Taylor até segunda ordem, da seguinte forma,

$$
\begin{aligned}
E\left[\delta n_{\mathbf{k}, \sigma}\right] & =E_{0}+\sum_{\mathbf{k}, \sigma} \epsilon_{\mathbf{k}} \delta n_{\mathbf{k}, \sigma}+ \\
+ & \frac{1}{2} \sum_{\mathbf{k} \mathbf{k}^{\prime}, \sigma, \sigma^{\prime}} f_{\mathbf{k} \sigma, \mathbf{k}^{\prime} \sigma^{\prime}} \delta n_{\mathbf{k}, \sigma} \delta n_{\mathbf{k}^{\prime}, \sigma^{\prime}}+O\left(\delta n^{3}\right)
\end{aligned}
$$

onde, em princípio, $\epsilon(\boldsymbol{k})$ e $f_{\mathbf{k} \sigma, \mathbf{k}^{\prime} \sigma^{\prime}}$ são funções arbitrárias do momento e spin. A equação 20 deve ser comparada com sua equivalente para o gás ideal, equação 12 , com a ressalva que a equação 12 é exata, enquanto que equação (20) é uma aproximação. Desta comparação vemos que, $E_{0}$ é a energia total do estado fundamental. A diferença do gás ideal de férmions, em relação ao caso do líquido de Fermi, é que não sabemos qual é seu valor. Cabe destacar que a teoria de Landau não tem entre seus objetivos predizer este valor, que apenas pode ser obtido com um cálculo microscópico. Continuando com a comparação, vemos que no caso em que $f_{\mathbf{k} \sigma, \mathbf{k}^{\prime} \sigma^{\prime}} \equiv 0$, as equações (12) e 20) são equivalentes. Podemos identificar então $\epsilon(\boldsymbol{k}) \equiv \epsilon^{0}(\boldsymbol{k})=|\boldsymbol{k}|^{2} / 2 m^{*}$. Apenas admitimos a massa efetiva na relação de dispersão. Ainda, por analogia com a equação (13), podemos escrever a relação de dispersão de uma quase-partícula como $\tilde{\epsilon}_{\sigma}(\mathbf{k})=\delta E / \delta n_{\mathbf{k}, \sigma}$, obtendo a partir da equação 20

$$
\tilde{\epsilon}_{\sigma}(\mathbf{k})=\epsilon_{\mathbf{k}}^{0}+\sum_{\mathbf{k}^{\prime}, \sigma^{\prime}} f_{\mathbf{k} \sigma, \mathbf{k}^{\prime} \sigma^{\prime}} \delta n_{\mathbf{k}^{\prime}, \sigma^{\prime}}
$$

Esta expressão exprime de forma mais evidente o conceito de quase-partícula. A relação de dispersão é afetada pelo segundo termo da equação 21, que representa a "nuvem" produzida pela deformação da distribuição de partículas $\delta n_{\mathbf{k}, \sigma}$ através da interação $f_{\mathbf{k} \sigma, \mathbf{k}^{\prime} \sigma^{\prime}}$. Desta forma, a função $f_{\mathbf{k} \sigma, \mathbf{k}^{\prime} \sigma^{\prime}}=\delta^{2} E / \delta n_{\mathbf{k}, \sigma} \delta n_{\mathbf{k}^{\prime}, \sigma^{\prime}}$ parametriza de forma geral as interações entre quase-partículas.

O fato de a energia total ser quadrática nos desvios $\delta n_{\mathbf{k}, \sigma}$, não deve ser interpretado como se a teoria fosse trivial. De fato, como dissemos anteriormente, as interações produzem uma variação da relação de dispersão dada pela equação 21. Por sua vez, a distribuição de quasepartículas vem dada pela distribuição de Fermi-Dirac, já que elas são férmions. Temos que,

$$
n_{\mathbf{k}, \sigma}=\frac{1}{1+e^{\left(\tilde{\epsilon}_{\sigma}(\mathbf{k})-\mu\right) / k_{B} T}}
$$

$\operatorname{com} \tilde{\epsilon}_{\sigma}(\mathbf{k})$ dado pela equação 21. Esta distribuição, por sua vez, volta a modificar o desvio $\delta n_{\mathbf{k}, \sigma}$ que acaba modificando finalmente $\tilde{\epsilon}_{\sigma}(\boldsymbol{k})$. Portanto, o cálculo de qualquer observável da teoria passa pela solução das equações 20, 21) e 22 de forma auto-consistente. Dessa forma, o fato das quase-partículas serem férmions, faz com que a simples expansão em serie de Taylor da energia total seja não trivial.

As equações 20, 21) e 22 são o coração da Teoria de Landau para líquidos de Fermi, fornecendo o andaime matemático que condensa os principais postulados da teoria.

\subsection{Parâmetros de Landau}

A função $f_{\mathbf{k} \sigma, \mathbf{k}^{\prime} \sigma^{\prime}}$, em princípio arbitrária, codifica todo nosso conhecimento da interação entre quase-partículas. Aplicando considerações de simetria podemos nos aprofundar na sua estrutura com o objetivo de formular modelos mínimos de interação.

Devido ao princípio adiabático de Landau, decorre que $f_{\mathbf{k} \sigma, \mathbf{k}} \sigma^{\prime}$ deve ser contínuo quando $\mathbf{k}$ ou $\mathbf{k}$ ' atravessar a 
superfície de Fermi. Caso contrário teríamos mudanças na interação entre quase-partículas quando estas saírem da superfície para estados desocupados. Como os desvios $\delta n(\boldsymbol{k})$ são concentrados sobre a superfície de Fermi, podemos ver a partir da equação 20 que é suficiente considerar a função calculada sobre a superfície de Fermi; isto é $f_{\mathbf{k}_{F} \sigma, \mathbf{k}_{F}^{\prime} \sigma^{\prime}}$.

Por um lado é evidente que a interação dever ser invariante perante permutações de $\boldsymbol{k}_{F} \leftrightarrow \boldsymbol{k}_{F}^{\prime}$. Por outro lado, se não houver um campo magnético aplicado, o sistema deveria ser invariante perante a tansformação $\sigma \rightarrow-\sigma$. Nessas condições existem apenas duas funções independentes, $f_{\mathbf{k}+, \mathbf{k}^{\prime}+}$ e $f_{\mathbf{k}+, \mathbf{k}^{\prime}}$. Onde o índice \pm se refere ao valor $\sigma= \pm 1 / 2$. É conveniente trabalhar com uma combinação linear dessas funções da seguinte forma,

$$
\begin{aligned}
& f_{\mathbf{k}, \mathbf{k}}^{s},=f_{\mathbf{k}+, \mathbf{k}^{\prime}+}+f_{\mathbf{k}+, \mathbf{k}^{\prime}-} \\
& f_{\mathbf{k}, \mathbf{k}}^{a},=f_{\mathbf{k}+, \mathbf{k}^{\prime}-}-f_{\mathbf{k}+, \mathbf{k}^{\prime}-}
\end{aligned}
$$

sendo que os sobre-índices $s$ e $a$ se referem as propriedades "simétrica" e "antisimétrica" em relação à transformação $\sigma \rightarrow-\sigma$. Dessa forma, a função simétrica $f_{\mathbf{k}, \mathbf{k}}^{s}$, representa a interação dos graus de liberdade de carga $\delta n^{s}=\delta n_{+}+$ $\delta n_{-}$, enquanto que a função anti-simétrica $f_{\mathbf{k}, \mathbf{k}}^{a}$, acopla com os graus de liberdade de spin $\delta n^{s}=\delta n_{+}-\delta n_{-}$.

Como estamos considerando um sistema isotrópico, isto é invariante perante rotações, as interações $f_{\mathbf{k}_{F}, \mathbf{k}_{F}^{\prime}}^{s(a)}$, não dependem do $\left|\boldsymbol{k}_{F}\right|=\left|\boldsymbol{k}_{F}^{\prime}\right|$, mas do ângulo entre os dois vetores $\boldsymbol{k}_{F} \cdot \boldsymbol{k}_{F}^{\prime}=\cos \theta$. Desta forma, as funções de interação podem ser representadas como séries em polinômios de Legendre,

$$
f_{\mathbf{k k}}^{s(a)}=\sum_{\ell=0}^{\infty} f_{l}^{s(a)} P_{\ell}(\cos \theta)
$$

onde $P_{\ell}(\cos \theta)$ são polinômios de Legendre de ordem $\ell$ e $f_{\ell}^{s(a)}$, com $\ell=0,1, \ldots$, são coeficientes arbitrários. Dessa forma, a interação fica completamente parametrizada pelo valor dos coeficientes $\left\{f_{\ell}^{s(a)}\right\}$. A interpretação física destes coeficientes é muito simples. Eles representam constantes de acoplamento correspondentes a diferentes canais de momento angular. Por exemplo, o coeficiente $f_{0}^{s}$, representa uma interação isotrópica de carga. Já o coeficiente $\operatorname{com} \ell=1$ representa uma interação dipolar. $\ell=2$ representa uma interação quadrupolar e assim por diante.

É conveniente escrever estes coeficientes de forma adimensional

$$
F_{l}^{s(a)}=N\left(\epsilon_{F}\right) f_{l}^{s(a)}
$$

onde $N\left(\epsilon_{F}\right)$ é a densidade de estados $N\left(\epsilon_{F}\right)=$ $(1 / V) \sum_{\boldsymbol{k}} \delta\left(\epsilon(\boldsymbol{k})-\epsilon_{F}\right)$, sobre a superfície de Fermi. As quantidades $F_{l}^{s(a)}$ medem a intensidade da força de interação em diversos canais de momento angular, comparada com a energia cinética. Eles parametrizam completamente a interação e são chamados de parâmetros de Landau. Os parâmetros de Landau são as únicas constantes fenomenológicas da teoria e devem ser determinados experimentalmente para cada sistema particular. O cálculo desses parâmetros a partir de modelos microscópicos é bastante difícil, sendo que é possível calculá-los para alguns sistemas simples e de forma perturbativa.

\section{Distribuições não Homogêneas}

A teoria apresentada na sessão anterior descreve pequenas flutuações da distribuição de partículas no estado fundamental. Essas flutuações são homogêneas, isto é, são produzidas em todo o espaço simultaneamente. Dessa forma, estamos descrevendo propriedades de equilíbrio do líquido de Fermi. Poderíamos tentar generalizar a formulação anterior, para permitir flutuações locais, ou não homogêneas e dependentes do tempo, $\delta n_{\boldsymbol{k}, \boldsymbol{\sigma}}(\boldsymbol{r}, t)$. Dessa forma, seríamos capazes de descrever propriedades fora do equilíbrio termodinâmico, como por exemplo, propriedades de transporte, ou propagação de ondas no meio. Uma dificuldade evidente dessa formulação é que a existência de uma distribuição local de partículas $n_{\boldsymbol{k}_{F, \sigma}}(\boldsymbol{r}, t)$ parece implicar que deveríamos conhecer o momento $\boldsymbol{k}$ e a posição $\boldsymbol{r}$ simultaneamente. Sabemos que a mecânica quântica impede esse conhecimento devido ao principio de incerteza de Heisemberg. A resposta a esse questionamento se encontra nas escalas de distância e tempo onde essa formulação pode ser aplicada. Sabemos que as flutuações $\delta n_{\boldsymbol{k}, \boldsymbol{\sigma}}(\boldsymbol{r}, t)$ são concentradas fortemente para momentos $\boldsymbol{k} \sim \boldsymbol{k}_{F}$. Por este motivo, o momento de Fermi, $k_{F}$ nos fornece uma escala natural de momento e uma escala natural de comprimentos dada por $1 / k_{F}$. Formular uma distribuição de partículas no"espaço de fases", $\left(\boldsymbol{k}_{F}, \boldsymbol{r}\right)$, faz sentido apenas numa escala em que $|\boldsymbol{r}|>>1 / k_{F}$. A mesma coisa acontece com o tempo. A energia de Fermi $\epsilon_{F}$ nos fornece uma escala natural de energias ou uma escala natural de tempos $1 / \epsilon_{F}$. Portanto, na presente formulação aceitamos que queremos descrever variações temporais com $t>>1 / \epsilon_{F}$. Outra forma de representar flutuações não homogêneas da distribuição de partículas é considerar o espaço de Fourier onde

$$
\tilde{n}_{\boldsymbol{k}_{F}, \sigma}(\boldsymbol{q}, \omega)=\int d \boldsymbol{r} d t n_{\boldsymbol{k}_{F}, \sigma}(\boldsymbol{r}, t) e^{i(\omega t-\boldsymbol{q} \cdot \boldsymbol{r})}
$$

O regime apropriado onde essa formulação é válida é $|\boldsymbol{q}|<<\boldsymbol{k}_{\boldsymbol{F}}, \omega<<\epsilon_{F}$. Nesse sentido, a formulação nãohomogênea da teoria de Landau é necessariamente uma teoria semi-clássica.

Tendo estabelecido o regime em que a formulação em espaço de fases pode ser aplicada, desenvolvemos a teoria em completa analogia ao caso homogêneo. Considera-se uma perturbação estática na distribuição do número de partículas na forma

$$
n_{\mathbf{k}}(\mathbf{r})=n_{\mathbf{k}}^{0}+\delta n_{\mathbf{k}}(\mathbf{r})
$$

Por simplicidade na notação, estamos ignorando o número quântico de spin, $\sigma$. De qualquer forma, ele pode ser reintroduzido caso seja conveniente. Percebe-se que a 
perturbação é feita no estado fundamental homogêneo $n_{\mathbf{k}}^{0}$. Fazer a expansão da energia em serie de Taylor com essa perturbação conduz à seguinte equação:

$$
\begin{aligned}
E & =E_{0}+\sum_{\mathbf{k}} \int d^{2} \mathbf{r} \epsilon(\mathbf{k}, \mathbf{r}) \delta n_{\mathbf{k}}(\mathbf{r}) \\
& +\frac{1}{2} \sum_{\mathbf{k k}} \iint d^{2} \mathbf{r} d^{2} \mathbf{r}^{\prime} f\left(\mathbf{k r}, \mathbf{k}^{\prime} \mathbf{r}^{\prime}\right) \delta n_{\mathbf{k}}(\mathbf{r}) \delta n_{\mathbf{k}},\left(\mathbf{r}^{\prime}\right)+\ldots
\end{aligned}
$$

Note a semelhança com a equação 20 para o caso homogêneo.

Algumas considerações físicas simplificam muito essa expressão. Em primeiro lugar, consideramos que o sistema é invariante perante translações. Isto nos força a escolher $\epsilon(\mathbf{k}, \mathbf{r}) \equiv \epsilon_{\mathbf{k}}$ e $f\left(\mathbf{k r}, \mathbf{k}^{\prime} \mathbf{r}^{\prime}\right) \equiv f_{\mathbf{k}, \mathbf{k}},\left(\boldsymbol{r}-\boldsymbol{r}^{\prime}\right)$. Consideramos também interações de curto alcance, de forma que podese escrever $f_{\mathbf{k}, \mathbf{k}},\left(\boldsymbol{r}-\boldsymbol{r}^{\prime}\right)=f_{\mathbf{k}, \mathbf{k}}, \delta\left(\boldsymbol{r}-\boldsymbol{r}^{\prime}\right)$, onde $\delta\left(\boldsymbol{r}-\boldsymbol{r}^{\prime}\right)$ é a delta de Dirac. A equação 29) adquire a forma mais compacta

$$
E=E_{0}+\int d^{2} \mathbf{r} \delta E(\mathbf{r})
$$

onde

$$
\delta E(\mathbf{r})=\sum_{\mathbf{k}} \epsilon_{\mathbf{k}} \delta n_{\mathbf{k}}(\mathbf{r})+\frac{1}{2} \sum_{\mathbf{k k}} f_{\mathbf{k k}}, \delta n_{\mathbf{k}}(\mathbf{r}) \delta n_{\mathbf{k}},(\mathbf{r})
$$

Em completa analogia com o caso homogêneo, definimos a energia de uma quase -partícula $\tilde{\epsilon}_{\boldsymbol{k}}(\boldsymbol{r})=\delta E / \delta n_{\boldsymbol{k}}(\boldsymbol{r})$, obtendo,

$$
\tilde{\epsilon}_{\mathbf{k}}(\boldsymbol{r})=\epsilon_{\mathbf{k}}+\sum_{\mathbf{k}} f_{\mathbf{k k}}, \delta n_{\mathbf{k}},(\mathbf{r})
$$

O fato de termos conseguido uma expressão local para a energia de uma quase-partícula ou, ainda, para a energia total, decorre do fato de ter considerado interações de curto alcance.

\subsection{A Equação de Transporte}

Tendo formulado uma expressão para a energia local, resta introduzir a variável temporal mediante uma equação dinâmica. Considerando o líquido de Fermi como um gás de quase-partículas fracamente interagente, desenvolvemos uma teoria cinética para o sistema.

Continuando com a perspetiva semi-clássica, podemos considerar a energia local de uma quase-partícula $\tilde{\epsilon}_{\mathbf{k}}(\boldsymbol{r})$ como um Hamiltoniano clássico no espaço de fases $(\boldsymbol{k}, \boldsymbol{r})$, dado pela equação (32). Utilizando o bem conhecido procedimento da teoria cinética [3], ou seja, considerando um pequeno volume do espaço de fase $d^{3} \mathbf{k} d^{3} \mathbf{r}$ e calculando o fluxo por cada lado desse elemento, obtém-se a equação que governa o fluxo de quase-partículas no espaço de fase, na ausência de colisões 7 :

$$
\frac{\partial n_{\mathbf{k}}(\boldsymbol{r}, t)}{\partial t}+\left\{n_{\mathbf{k}}(\mathbf{r}, t), \tilde{\epsilon}_{\mathbf{k}}(\boldsymbol{r})\right\}=0
$$

onde $\{$,$\} são os parênteses de Poisson. Desenvolvendo$ explicitamente o parênteses de Poisson, tem-se:

$$
\frac{\partial n_{\mathbf{k}}}{\partial t}+\nabla_{\mathbf{r}} n_{\mathbf{k}} \cdot \nabla_{\mathbf{k}} \tilde{\epsilon}_{\mathbf{k}}-\nabla_{\mathbf{k}} n_{\mathbf{k}} \cdot \nabla_{\mathbf{r}} \tilde{\epsilon}_{\mathbf{k}}=0
$$

Essa equação é uma equação de Boltzman, onde temos ignorado a integral de colisões. Vemos que as fontes de variação temporal tem duas origens. O coeficiente do segundo termo da equação (34), proporcional à inomogeneidade, pode ser identificado como a velocidade de Fermi quando projetado sobre a superfície de Fermi,

$$
\boldsymbol{v}_{F}=\left.\nabla_{\mathbf{k}} \tilde{\epsilon}_{\mathbf{k}}\right|_{\boldsymbol{k}=\boldsymbol{k}_{F}}
$$

Por outro lado, no terceiro termo da equação (34), aparece uma força difusiva, $-\nabla_{\mathbf{r}} \tilde{\epsilon}_{\mathbf{k}}$ que tende a puxar as quase-partículas perturbadas de volta às regiões de mínima energia.

Devido a que a energia depende da função distribuição de partículas, a equação (34) é altamente não linear. Uma equação simplificada pode ser obtida usando a equação (28) e linearizando o resultado em termos da flutuação $\delta n_{\boldsymbol{k}}(\boldsymbol{r}, t)$. Obtemos,

$$
\begin{aligned}
\frac{\partial \delta n_{\mathbf{k}}(\mathbf{r}, t)}{\partial t} & +\mathbf{v}_{\mathbf{k}} \cdot \nabla_{\mathbf{r}} \delta n_{\mathbf{k}}(\mathbf{r}, t) \\
& -\nabla_{\mathbf{k}} n_{\mathbf{k}}^{0} \cdot \sum_{\mathbf{k}} f_{\mathbf{k k}}, \nabla_{\mathbf{r}} \delta n_{\mathbf{k}},(\mathbf{r}, t)=0
\end{aligned}
$$

Onde usamos o fato que $\delta n_{k}$ é fortemente concentrada para valores de $|\boldsymbol{k}| \sim k_{F}$ e a velocidade de Fermi vem dada pela equação 35 . Note também que $\nabla_{\mathbf{k}} n_{\mathbf{k}}^{0}=$ $\mathbf{v}_{\mathbf{k}} \delta\left(\epsilon_{\mathbf{k}}-\epsilon_{F}\right)$ o que faz que em toda a equação os valores de $\boldsymbol{k}$ estejam sobre a superfície de Fermi.

A interpretação física da equação 36 é clara. A primeira linha descreve a evolução das excitações de quasepartículas livres. Note que a evolução se dá na mesma direção que a velocidade de Fermi. Para nosso caso isotrópico, é uma evolução radial. Já a segunda linha leva em conta as interações dadas pela função $f_{\boldsymbol{k}, \boldsymbol{k}^{\prime}}$ que pode ser eventualmente escrita em termos dos parâmetros de Landau.

$\mathrm{Na}$ próxima sessão usaremos a equação (36), para descrever excitações coletivas num modelo simples de líquido de Fermi.

\section{Excitações coletivas}

No geral, se tirarmos localmente o sistema fora do equilíbrio, existem forças restauradoras que tendem a levar o sistema novamente ao equilíbrio. Porém, poderia acontecer que algum tipo de excitação, como a propagação de certas ondas no fluido, pudesse ser propagada sem dissipação. Este tipo de excitação seria análoga à propagação de fônons, ou "som" num meio.

Para explorar esse tipo de excitação, podemos procurar soluções da equação (36) em algum tipo de condição específica. Suponha que procuramos soluções do tipo,

$$
\delta n_{\mathbf{k}}(\mathbf{r}, t)=\delta n_{\mathbf{k}}(\mathbf{q}, \omega) e^{i(\mathbf{q} \cdot \mathbf{r}-\omega t)}
$$


Esta flutuação representa uma onda de frequência $\omega$ e vetor de onda $\boldsymbol{q}$. A pergunta relevante é se existe alguma solução da equação (36), para algum valor de $\omega, \boldsymbol{q}$. Substituindo a equação (37) na equação (36) obtemos,

$$
\left(\mathbf{q} \cdot \mathbf{v}_{\mathbf{k}}-\omega\right) \delta n_{\mathbf{k}}-\mathbf{q} \cdot \mathbf{v}_{\mathbf{k}}\left(\frac{\partial n_{\mathbf{k}}^{0}}{\partial \epsilon_{\mathbf{k}}}\right) \sum_{\mathbf{k}} f_{\mathbf{k k}}, \delta n_{\mathbf{k}},=0
$$

Para avançar na solução dessa equação temos que especificar um "modelo" de líquido de Fermi. Isto significa fixar a interação e a dimensionalidade. Por simplicidade, com o intuito de fornecer o exemplo mais simples possível, escolhemos um sistema bidimensional, e uma interação isotrópica. Isto é $f_{\boldsymbol{k}, \boldsymbol{k}^{\prime}} \equiv f_{0}$. Isto é equivalente a escolher o parametro de Landau $f_{0}^{(s)} \neq 0$, sendo que $f_{\ell}^{s(a)}=0$ para $\ell=1,2, \ldots$. Ainda, devido ao fato da flutuação estar fortemente concentrada na superfície de Fermi, podemos parametrizá-la como

$$
\delta n_{\mathbf{k}}(\omega, \boldsymbol{q})=\delta\left(\epsilon_{\mathbf{k}}-\epsilon_{F}\right) v_{F} \nu(\omega, \boldsymbol{q}, \theta)
$$

A função delta de Dirac, exprime o fato de o momento $\boldsymbol{k}$ estar sobre a superfície de Fermi. Desta forma, a função $\nu(\omega, \boldsymbol{q}, \theta)$ representa a perturbação no número de partículas com frequência $\omega$, vetor de onda $\boldsymbol{q}$, propagando-se na direção $\theta$, definida como $\mathbf{q} \cdot \mathbf{v}_{F}=\cos \theta$. Com esta definição do modelo e a parametrização da equação 39 , a equação 38 toma a forma mais simples,

$$
\begin{aligned}
\left(\cos (\theta)-\frac{\omega}{q v_{F}}\right) & \nu(\omega, \boldsymbol{q}, \theta) \\
& -F_{0} \cos (\theta) \int_{0}^{2 \pi} d \theta^{\prime} \nu\left(\omega, \boldsymbol{q}, \theta^{\prime}\right)=0
\end{aligned}
$$

onde $q=|\boldsymbol{q}|$ e o parâmetro de Landau adimensional $F_{0}=N\left(\epsilon_{F}\right) f_{0}$ como descrito na sessão anterior. Podemos observar várias características dessa equação. Em primeiro lugar é uma equação linear homogênea. Portanto, $\nu(\omega, \boldsymbol{q}, \theta) \equiv 0$, sempre é uma solução. A existência de outras soluções vai depender de valores específicos para $\omega, \boldsymbol{q}$. A equação 40 é uma equação algébrica na frequência e no vetor de onda, e depende apenas da combinação adimensional $s=\omega / v_{F} q$. A variável $s$ tem uma interpretação física bem interessante; é uma relação entre duas energias. O numerador é a energia associada a propagação de uma onda com frequência $\omega$, enquanto que o denominador $v_{F} q$ é a energia de uma excitação partícula-buraco com momento $\boldsymbol{q}$. Finalmente, tem que ser observado que a equação 40 é uma equação integral na variável $\theta$ que parametriza o ângulo relativo entre o vetor de onda $\boldsymbol{q}$ e a velocidade de Fermi $\boldsymbol{v}_{F}$.

Concluímos portanto que a solução deve ter a forma $\nu(\omega, \boldsymbol{q}, \theta)=\nu(s, \theta)$. Observando a segunda linha da equação 40, vemos que a última integral só pode ser uma função $s$,

$$
C(s)=\int_{0}^{2 \pi} d \theta \nu(\omega, \boldsymbol{q}, \theta)
$$

Resolvendo a equação 40 em termos de $C(s)$, obtemos,

$$
\nu(s, \theta)=F_{0} C(s) \frac{\cos \theta}{\cos \theta-s}
$$

onde $C(s)$ ainda deve ser determinado. Porem, observamos que se integrarmos em $\theta$ ambos lados da equação (42) obtemos,

$$
\int_{0}^{2 \pi} d \theta \frac{\cos \theta}{\cos \theta-s}=\frac{1}{F_{0}}
$$

que é uma condição para a variável $s$. Isto é, a solução (42) só pode existir para os valores de $s$ que satisfazem a equação 43 . Integrando essa equação obtemos um único valor de $s$ dado por,

$$
s=\frac{\omega}{v_{F} q}=\frac{1+F_{0}}{\sqrt{\left(1+F_{0}\right)^{2}+F_{0}}}
$$

Portanto, concluímos que o presente modelo suporta propagação de ondas, desde que a dispersão seja linear, $\omega=V_{0} q$, e a velocidade de propagação seja

$$
V_{0}=\frac{1+F_{0}}{\sqrt{\left(1+F_{0}\right)^{2}+F_{0}}} v_{F}
$$

Observamos que, para interações repulsivas, para as quais $F_{0}>0$, o líquido pode propagar ondas sem dissipação com $V_{0}<v_{F}$. Note que para $F_{0} \rightarrow 0, V_{0} \rightarrow v_{F}$, desde que a única escala de velocidades do sistema livre seja a velocidade de Fermi. Este modo de propagação foi calculado pela primeira vez por Landau em um modelo tridimensional e foi chamado (em inglês) de "zero sound".

Uma análise da equação de transporte, mais detalhada, com modelos de interação um pouco mais complexos, pode ser encontrado na ref. [11].

\section{Conclusão}

Neste artigo tentamos apresentar uma introdução à Teoria de Landau para Líquidos de Fermi da forma mais simples e didática possível.

Primeiramente, revimos o conceito de gás de Fermi e sua formulação em termos de número de ocupação. É interessante notar que o princípio de exclusão de Pauli é o único conceito necessário para entender as principais propriedades desse sistema, sem necessidade de recorrer à mecânica estatística quântica.

As principais ideias de Landau para incorporar o efeito de interações foram abordadas de forma qualitativa, para logo depois ser apresentado o embasamento matemático para implementar estas ideias. Finalmente, mostramos um exemplo de propagação de ondas em um modelo simplificado de líquido de Fermi que, embora seja muito simples, permite obter resultados não triviais. É de bom tom apontar que o ponto chave no desenvolvimento dessa teoria é a interpretação que Landau teve da natureza, formulando hipóteses não elementares que mais tarde foram 
comprovadas, ao invés da construção de um arcabouço matemático abstrato.

Acreditamos que este tipo de abordagem é completamente viável nos cursos de graduação, no mesmo nível que outros assuntos abordados tradicionalmente num curso de estrutura da matéria. Defendemos que é essencial focar no papel que a Teoria de Landau teve no desenvolvimento epistemológico da área, onde seu salto conceitual foi notável, pois estabeleceu todo um paradigma, sendo fundamental para o entendimento de outros fenômenos como a supercondutividade e a superfluidez que tem tido e tem um importante destaque na física moderna e contemporânea.

\section{Agradecimentos}

Este trabalho foi financiado em parte pelo CNPq, FAPERJ e CAPES.

\section{Referências}

[1] R.F. Streater e A.S. Wightman, PCT, Spin and Statistics, and All that (Princeton University Press, Princeton, 2000).

[2] W. Pauli, Phys. Rev. 58, 716 (1940).

[3] K. Huang, Statistical Mechanics (John Wiley and Sons, New York, 1987).

[4] R.D. Mattuck, A Guide to Feynman Diagrams in the Many-body Problem (Dover Publications, New York, 1992).

[5] S.B. Dugdale, Physica Scripta 91, 53009 (2016).

[6] L. Landau, Soviet Physics Journal of Experimental and Theoretical Physics 3, 920 (1957).

[7] G. Baym e C. Pethick, Landau Fermi-Liquid Theory: Concepts and Applications (John Wiley and Sons, New York, 1991).

[8] D. Pines e P. Nozières, The Theory of Quantum Liquids (Avalon Publishing, New York, 1999).

[9] J.M. Luttinger, Phys. Rev. 119, 1153 (1960).

[10] A.B. Migdal, Journal of Experimental and Theoretical Physics 5, 333 (1957).

[11] R. Aquino e D.G. Barci, Phys. Rev. B 100, 115117 (2019). 